\title{
Paradiplomacia local y transfronteriza como un instrumento de gobernanza ambiental en el Mercosur y la Unión Europea

\author{
Una descripción comparada
}

Paradiplomacia local e transfronteiriça como um instrumento de governança ambiental no Mercosul e na União Europeia

Uma descrição comparada

\section{Local cross-border paradiplomacy as an environmental governance tool in Mercosur and the European Union}

A comparative approach

\author{
Nahuel Oddone* \\ Horacio Rodríguez Vázquez** \\ Martín J. Quiroga Barrera Oro***
}

\begin{abstract}
Resumen: La complejidad y expansión de los problemas ambientales han hecho que el estado nación haya perdido su exclusividad en la toma de decisiones en la materia. Actualmente múltiples actores y agencias contribuyen con la gobernanza ambiental destacándose, muy especialmente, los aportes de los gobiernos locales a partir de su paradiplomacia. En este artículo se entiende que la paradiplomacia es una herramienta que permite hacer operativa la gobernanza ambiental. Los gobiernos suelen enfrentar dificultades técnico-científicas y financieras a la vez que presentan oportunidades por su proximidad con la ciudadanía y su mejor conocimiento territorial. A modo de ejemplo, se presentan dos experiencias que dan cuenta de cómo la paradiplomacia, tanto en procesos de integración (la Unidad Temática de Ambiente y Desarrollo Sostenible de la Red de Mercociudades y el Pacto de Alcaldes contra el Cambio Climático de la Unión Europea) como a través de la cooperación transfronteriza, han contribuido con la gestión y gobernanza ambiental.
\end{abstract}

Palabras clave: Paradiplomacia ambiental. Gobernanza ambiental. Red de Mercociudades. Pacto de Prefeitos contra el Cambio Climático. Cooperación transfronteriza.

\footnotetext{
* Centro Regional de Promoción de la Mipyme (Cenpromype) del Sistema de la Integración Centroamericana (Sica) (San Salvador, El Salvador).

** The Nature Conservancy (Ciudad de México, México).

*** Banco Interamericano de Desarrollo (Washington D.C., Estados Unidos).

autores.datos_biográficos
}

Civitas, Porto Alegre, v. 18 , n. 2, p. 332-350, mayo-agosto 2018 
Resumo: A complexidade e expansão dos problemas ambientais têm feito com que o estado nação tenha perdido sua exclusividade na tomada de decisões na matéria. Atualmente múltiplos atores e agências contribuem para a governança ambiental destacando-se, especialmente, as contribuições dos governos locais a partir de sua paradiplomacia. Neste artigo se entende que a paradiplomacia é uma ferramente que torna a governança ambiental operativa. Os governos costumam enfrentar dificuldades técnico-científicas e financeiras ao mesmo tempo em que apresentam oportunidades por sua proximidade com a cidadania e seu melhor conhecimento territorial. Por exemplo, se apresentam duas experiências que dão conta de como a paradiplomacia, tanto em processos de integração (a Unidade Temática de Ambiente e Desenvolvimento Sustentável da Rede Mercocidades e o Pacto de Prefeitos contra a Mudança Climática da União Europeia) como através da cooperação transfronteiriça, têm contribuído para a gestão e governança ambiental.

Palavras-chave: Paradiplomacia ambiental. Governança ambiental. Rede Mercocidades. Pacto de alcades contra a mudança climática. Cooperação transfronteiriça.

\begin{abstract}
The complexity and expansion of environmental issues have caused the state to lose its decision-making exclusivity in this area. Currently multiple actors and agencies contribute to environmental governance, especially in the realm of local governance thanks to their paradiplomacy. In this article, paradiplomacy is understood as a tool that allows operationalizing environmental governance. Governments often face technical, scientific and financial difficulties but at the same time have opportunities due to their proximity to their citizens and better territorial knowledge. As an example, two experiences are presented that serve to prove how paradiplomacy has contributed to environmental governance and management, as much as in cities nets related to integration processes' (the Unidad Temática Ambiental y Desarrollo Sostenible of the Mercociudades Network and the Covenant of Mayors against the Climate Change of the European Union) as well as through the cross border cooperation.
\end{abstract}

Keywords: Environmental paradiplomacy. Environmental governance. Mercociudades network. Covenant of Mayors against the Climate Change. Crossborder cooperation.

\title{
Introducción
}

El equilibrio climático constituye un continuum ecológico que se extiende tanto por los espacios sometidos a soberanía territorial como más allá de ellos (Ruiz, 1999). Los impactos de los problemas ambientales son y deben ser entendidos en el largo plazo con sus afectaciones a nivel global, regional, nacional y local (Bodansky, 1999, Cardozo et al., 2014; Oddone y Rodríguez, 2014). Esta particularidad de los problemas ambientales globales ha hecho que, de formar parte exclusiva de la agenda internacional de los estados, en la última década, nuevos actores de la arena internacional se fueran incorporando en las respuestas de mitigación y las propuestas de adaptación, ya sean actores del sector privado y de la sociedad civil o las esferas "internas" 
de los estados que reclaman por su inserción en los nuevos modelos de gobernanza ambiental. ${ }^{1}$

La creciente participación de los gobiernos subnacionales en la gobernanza ambiental global responde a la combinación de factores externos (globalización e interdependencia, el incremento en la frecuencia e intensidad de eventos extremos vinculados al cambio climático, los acuerdos multilaterales ambientales, la Agenda 21 y el debate mundial sobre el desarrollo sustentable) e internos (procesos de democratización, descentralización, desconcentración y reformas estructurales, así como reformas constitucionales). Dicha intervención se ha dado, sobre todo, a través de mecanismos de cooperación internacional (descentralizada y/o transfronteriza) y la conformación de redes de gobiernos locales que promueven la sustentabilidad y la protección del medio ambiente (Rodríguez y Lucatello, 2013). La globalización y la urgencia de hacer frente a los problemas ambientales globales, principalmente el cambio climático, han fomentado una mayor participación de los gobiernos subnacionales en la gobernanza ambiental global. Esta intervención se ha dado, sobre todo, a través de mecanismos de cooperación internacional (descentralizada y/o transfronteriza) y la conformación de redes de gobiernos locales que promueven la sustentabilidad y la protección del medio ambiente.

En este contexto, la paradiplomacia ${ }^{2}$ aparece como una herramienta clave para que los gobiernos subnacionales fortalezcan sus capacidades de protección ambiental; así como de prevención, adaptación y mitigación al cambio climático, sean más efectivos en la promoción de la sustentabilidad a nivel local y contribuyan en la provisión de bienes y servicios ambientales. De igual manera, la paradiplomacia resalta la importancia de la articulación de los gobiernos locales en el Régimen Internacional del Cambio Climático, la economía verde, la seguridad ambiental y la gestión de bienes públicos regionales y/o globales, entre otros aspectos. Derivado de lo anterior, es posible

\footnotetext{
${ }^{1}$ A modo de ejemplo, en la COP 19, desarrollada en Varsovia entre el 11 y el 22 noviembre de 2013, se instauró el "Día de las ciudades" como señal del vínculo entre cambio climático y gobiernos locales. A partir de ahora, el 21 de noviembre de cada año se festejará el día de las ciudades, creado a partir de una iniciativa conjunta entre la Presidencia de la COP, la Secretaría del Convención Marco de las Naciones Unidas sobre Cambio Climático, la Ciudad de Varsovia y el Iclei-Gobiernos Locales por la Sustentabilidad.

${ }^{2}$ El término paradiplomacia hace alusión a la acción exterior de los gobiernos subnacionales; es decir, aquellas acciones en el plano internacional que son protagonizadas por los gobiernos no centrales reconociéndose, por lo general, que las mismas están orientadas a la promoción del desarrollo local/territorial. El ejercicio de la actividad paradiplomática se da mediante el establecimiento de contactos permanentes o ad hoc con entidades públicas o privadas extranjeras, con el propósito de promover diversos aspectos socioeconómicos o culturales, así como cualquier otra dimensión exterior del sector público subnacional dentro de sus competencias constitucionales (Cornago, 2001).
} 
afirmar que gobernanza ambiental global y paradiplomacia son dos conceptos estrechamente vinculados, dado que el segundo permite la instrumentación del primero.

La gobernanza, en un sentido amplio, implica "la asignación autoritaria de recursos y el ejercicio del control y la coordinación, en donde los actores gubernamentales no son necesariamente los únicos participantes ni los más importantes" (Bulkeley, 2005, p. 877). Una gobernanza efectiva requiere establecer un marco ampliamente aceptado que permita institucionalizar la interacción entre grupos interesados, negociar los intereses contrapuestos y mitigar los conflictos, para determinar así la forma en que se llevarán a cabo la toma de decisiones y los acuerdos para el ejercicio del poder (Leeuwis y Van den Ban, 2004; Brenner, 2010). Esta fragmentación de las decisiones e intereses, influenciada por los estudios burocráticos y organizacionales, ha contribuido con el desarrollo de una serie de estudios que fortalecen el papel de la paradiplomacia en materia de gobernanza ambiental ${ }^{3}$ y se encuadran dentro de lo que Miriam Alfie Cohen (2013) denomina la visión pluralista de la gobernanza ambiental.

La visión pluralista pretende evaluar la formulación y la eventual efectividad de las políticas ambientales. La premisa guía de esta escuela considera a la política pública como resultado de intereses competitivos y recursos limitados. El papel de las instituciones estatales será proveer un marco justo para la toma de decisiones. Para estos teóricos, la pregunta clave consiste en establecer cómo las instituciones gubernamentales agregan múltiples intereses competitivos del campo ambiental para producir políticas efectivas. Este enfoque se centra en las prácticas estatales y, aunque la problemática ambiental y las metas por alcanzar han provocado serios conflictos al interior del aparato de estado, se busca edificar pactos y consensos en la construcción de políticas ambientales (Alfie Cohen, 2013). La aproximación presentada en este artículo se encuadra dentro de esta visión pluralista que es complementada por los aportes sobre la gestión de redes de Rhodes (1997). Este autor entiende la gobernanza como una forma de auto-organización de redes

\footnotetext{
3 "Los principales estudios sobre gobernanza ambiental estuvieron comandados por la ciencia política. Desde este ámbito, se cuestionaba las condiciones de formulación y la efectiva puesta en práctica de la gobernanza ambiental en las estructuras burocráticas existentes. En contraste, recientes estudios de la sociología ambiental como la sociedad del riesgo, la modernización ecológica y la llamada sociedad mundial emplean altos niveles de abstracción para teorizar procesos macrosociales y definir los vínculos globales-locales de la problemática ambiental" (Alfie Cohen, 2011, p. 16). Estos enfoques teóricos se concentraron en entender el papel que desempeña el estado, y su estructura interna, en materia de mitigación y adaptación al cambio climático como problema fundamental de la gobernanza ambiental.
} 
inter-organizacionales interdependiente que trabajan con un mismo interés bajo el intercambio de recursos, reglas reconocidas por sus miembros y una autonomía significativa frente al estado.

El presente artículo se divide en una introducción en la que se han plasmado los principales lineamientos teóricos, tres secciones y unas conclusiones. En la primera sección se analiza la paradiplomacia como parte de una gestión ambiental articulada. En la segunda se presentan dos experiencias de paradiplomacia ambiental en el marco de la integración regional para fortalecer la gobernanza ambiental, es decir, la Unidad Temática de Ambiente y Desarrollo Sustentable de la Red de Mercociudades y el Pacto de Alcaldes contra el Cambio Climático de la Unión Europea. En la tercera sección, se estudia la importancia de la cooperación transfronteriza como forma de paradiplomacia y como estrategia para una gestión ambiental regional. Por último, se extraen algunas conclusiones.

\section{La paradiplomacia como parte de una gestión ambiental articulada}

La gestión ambiental incluye las directrices, lineamientos, políticas y acciones específicas en materia de uso, aprovechamiento y conservación del medio ambiente y los recursos naturales desde una perspectiva que promueva el equilibro entre los tres pilares de la sustentabilidad: social, económico y ambiental.

En ese entendido, la gestión ambiental puede analizarse desde diferentes niveles: local, nacional, regional y global. En lo que respecta al nivel de análisis local, la importancia de los gobiernos subnacionales es clave. En primer lugar, constituyen una plataforma para el diseño de políticas públicas ambientales desde abajo, no sólo por su mayor proximidad a la ciudadanía y la posibilidad de tener una mejor comprensión de sus demandas sino también por poseer un conocimiento territorial más profundo, pudiendo dar cuenta de las amenazas o retos que enfrentan sus comunidades. En segundo término, los efectos del cambio climático son mayores a nivel local, por lo que los gobiernos subnacionales son clave en cuestiones de prevención, adaptación y mitigación.

Por otro lado, los gobiernos subnacionales, apoyados por herramientas como la paradiplomacia, juegan un papel cada vez más relevante en la gestión ambiental a nivel nacional, regional y global. Son actores fundamentales en la gestión compartida y articulada de los bienes públicos regionales/globales, por ejemplo, en la cooperación transfronteriza respecto de cuencas hídricas o zonas naturales protegidas. Asimismo, contribuyen con la visibilidad internacional 
de las políticas y programas públicos de desarrollo local sustentable, a través de su articulación en redes como Gobiernos Locales por la Sustentabilidad (Iclei) ${ }^{4}$ o la Red de Gobiernos Regionales para el Desarrollo Sostenible (nrg4SD). ${ }^{5}$ Asimismo, la participación de los gobiernos subnacionales en la gestión ambiental se materializa con apoyo de la paradiplomacia en acciones de cooperación descentralizada, como los programas de desarrollo local sustentable y gestión de residuos sólidos urbanos de la Deutsche Gesellschaft für Internationale Zusammenarbeit o del Programa UR-BAL de la Comisión Europea.

Es importante destacar que a través de la actividad paradiplomática los gobiernos locales también pueden iniciar políticas de promoción de una marca ciudad como "ciudad verde" o de su compromiso con el medioambiente como las Resilient Cities. Es factible que, además, puedan captar inversión extranjera directa y otros flujos de capital tendientes a financiar obras que promuevan el desarrollo local sostenible como el grupo de Living Cities. También cabe mencionar la posibilidad de participación conjunta en ferias, eventos y/o giras internacionales de negocios en el marco de la economía verde y/o la promoción de la sustentabilidad con el apoyo de organizaciones sin fines de lucro como Next City.

Las acciones arriba mencionadas, entre otras, constituyen ejemplos de la contribución de los gobiernos locales, en general, y la paradiplomacia,

\footnotetext{
${ }^{4}$ Iclei es una organización internacional, no gubernamental, no lucrativa, que funciona como una asociación de gobiernos locales, fundada en 1990 en la sede de Naciones Unidas, en Nueva York, Estados Unidos. Es observadora oficial de las Convenciones sobre Cambio Climático, Diversidad Biológica y de Lucha contra la Desertificación. Tiene como misión construir y dar apoyo a un movimiento mundial para mejoras tangibles en las condiciones ambientales locales; así como generar condiciones para el desarrollo sustentable global, a través de acciones locales acumulativas. Actualmente, Iclei se encuentra en 86 países, con más de 1,300 socios, y representando a más de 850 millones de personas (Oddone y Rodríguez Vázquez, 2014).

${ }^{5}$ Es una red internacional que representa a asociaciones regionales y gobiernos regionales a nivel global. Promueve el entendimiento, la colaboración y las asociaciones en materia de desarrollo sostenible, particularmente en la agenda climática, persiguiendo un mayor reconocimiento internacional sobre la importancia que las contribuciones regionales suponen al desarrollo sostenible. Nrg4SD fue creada por ciertos gobiernos regionales participantes en la Cumbre Mundial sobre Desarrollo Sostenible de Johannesburgo (2002), en una conferencia paralela. De esta conferencia paralela resultó la Declaración de Gauteng, firmada por 23 gobiernos subnacionales, en la que de un lado se ponía de manifiesto la importancia de los gobiernos subnacionales en asuntos de desarrollo sostenible, así como de la cooperación entre sí; y de otro lado, se acordaba el deseo de crear una red de gobiernos subnacionales a nivel mundial para el desarrollo sostenible (Declaración de Gauteng, 2002). A partir de la Conferencia de San Sebastián (2003) se inicia la personalización jurídica de la red, que se registra legalmente en 2004 como una "Asociación Internacional sin ánimo de lucro", bajo la ley belga. En la actualidad, la red cuenta con 50 miembros de derecho, de 30 países y 7 asociaciones de regiones, además de observadores, representando a más de mil gobiernos regionales a nivel mundial. $\mathrm{Nrg} 4 \mathrm{SD}$ es miembro del Foro Global de Asociaciones de Regiones (Fogar) y su portavoz para los temas de desarrollo sostenible (Cardozo et al., 2014).
} 
en particular, en la gestión ambiental integral. No obstante, muchas de las administraciones locales enfrentan una serie de desafíos para desarrollar y, sobre todo, mantener una agenda de paradiplomacia ambiental, dada la baja profesionalización de sus recursos humanos o las restricciones presupuestarias.

Durante las últimas tres décadas algunos gobiernos subnacionales han hecho uso de la paradiplomacia para atender aspectos que tradicionalmente no formaban parte de sus competencias, como la gestión ambiental, a través de la cooperación descentralizada y su participación en redes o asociaciones internacionales de gobiernos locales. De esta forma se ha estimulado una distribución de competencias en materia ambiental entre el gobierno central y los gobiernos locales, implementando estrategias de desarrollo que, al menos en la letra, parten de una planeación participativa (Ponce, 2010) entre diferentes niveles de gobierno y actores territoriales, contribuyendo al fortalecimiento de la gobernanza ambiental.

Sin embargo, la operatividad de dichas estrategias ha enfrentado obstáculos importantes a nivel estatal o provincial $\mathrm{y}$, más aún, municipal. A grandes rasgos, los gobiernos subnacionales se caracterizan por una débil capacidad técnica ${ }^{6}$ para iniciar $y$, sobre todo, mantener una agenda paradiplomática, debido a que la incursión de los gobiernos subnacionales en el ámbito exterior no necesariamente está acompañada de un marco jurídico-institucional, de recursos financieros adicionales, o de los conocimientos y habilidades técnicas para llevar a cabo el diseño y la instrumentación de estrategias de proyección internacional vinculadas al desarrollo local (Rodríguez y Lucatello, 2013; Rodríguez, Lucatello y Oddone, 2014).

Lo anterior se ha traducido en una falta de voluntad política de los gobernadores y los presidentes municipales sobre los asuntos internacionales, que muchas veces perciben que este tipo de actividades no les ofrece beneficios directos (no comportan votos) o las ubican como un gasto y no como una inversión (Velázquez y Schiavon, 2010). A esto se le añade la visión

\footnotetext{
${ }^{6}$ De acuerdo con Esther Ponce, la capacidad técnica de un gobierno subnacional se considera desde tres ámbitos diferentes: el plano político-institucional, la estructura administrativa y la relación del gobierno subnacional con la sociedad. El plano político-institucional hace referencia a las relaciones existentes entre la federación y los gobiernos locales, así como al sistema de partidos que predomina, lo que pone de relieve las relaciones de poder y los estilos de conducción de las políticas. La estructura administrativa permite evaluar el tipo de estructura orgánica-funcional que predomina en el gobierno subnacional; es decir, las competencias asignadas a cada área, la circulación real de la información y las tareas que logra cumplir el plantel subnacional. La relación del gobierno subnacional con la sociedad aparece como el lugar en donde se visualiza el tipo de gestión que efectivamente predomina en el gobierno local; así como la capacidad de apertura y articulación con otras instituciones y organizaciones de la sociedad: organizaciones de la sociedad civil (OSC), medios de comunicación etc. (Ponce, 2010).
} 
de corto plazo que generalmente caracteriza a las autoridades locales (Macht, 2010), dificultando entonces la institucionalización de este tipo de acciones. La baja institucionalidad per se de las oficinas o coordinaciones de asuntos internacionales de los gobiernos subnacionales, en muchos casos, termina repercutiendo en que el trabajo efectuado por una administración no sea retomado por el gobierno sucesor (Schiavon y Velázquez, 2009), llevando a una discontinuidad de políticas entre una administración y otra.

Esta discontinuidad también puede verse ocasionada por aspectos presupuestarios. Las unidades subnacionales suelen enfrentar problemas de financiamiento, en parte, por su relativamente baja capacidad de recaudación fiscal, lo que no les permite contar con los recursos financieros necesarios para poder instrumentar las políticas ambientales pertinentes o, una vez ejecutadas, asegurar su sostenibilidad en el tiempo. Asimismo, hay un papel poco relevante de la gestión internacional en la agenda de algunas asociaciones de gobiernos locales, pues se entiende como algo escindido de la realidad cotidiana y de las responsabilidades y tareas asignadas por la ley a dichos gobiernos. De acuerdo con Oostveen (2010), este tipo de obstáculos inciden en la consolidación de una paradiplomacia de calidad puesto que no se tiene registro de la relevancia que la gestión internacional reviste para que los gobiernos subnacionales, así como también sus redes o asociaciones, estén en condiciones de ejecutar mejor sus tareas y responsabilidades principales y que, en todo caso, la paradiplomacia puede ser una herramienta clave para el fortalecimiento de la autonomía de los gobiernos subnacionales, como ha ocurrido en algunos países de América Latina, en España y Portugal. ${ }^{7}$

A todas estas dimensiones se le suma la influencia de la ubicación geográfica de los estados y/o municipios. El territorio - que no puede ser entendido sin la acción humana y sus efectos sobre el mismo - se ve atravesado por las distintas dinámicas geopolíticas. De este modo, la topografía y los accidentes geográficos, aunado a la existencia o ausencia de acuerdos y/o conflictos políticos regionales o locales, son un catalizador importante de la paradiplomacia ambiental. Como se mencionó anteriormente, la geopolítica cobra mayor relevancia inclusive si los gobiernos subnacionales limitan con

\footnotetext{
${ }^{7}$ En este marco resulta interesante la experiencia del Programa Local de Adaptación al Cambio Climático lanzado por la Red Argentina de Municipios frente al Cambio Climático (RAMCC), la Cumbre Amazónica de los Gobiernos Locales por el Cambio Climático convocada por la Confederación Nacional de Municipios de Brasil (CNM) y la Asociación de Municipios Amazónicos (AAM), los Planes de Acción Climática Municipal en México con el respaldo técnico del Iclei o la Estrategia Local de Cambio Climático ha sido elaborada por la Red Española de Ciudades por el Clima, como Sección de la Federación Española de Municipios y Provincias (FEMP).
} 
otros países; por el mero hecho de su localización, dichos municipios son más proclives a ejecutar acciones de cooperación transfronteriza.

La consolidación de la paradiplomacia para la gobernanza ambiental depende de diversos factores, entre los que se destacan: 1) la capacidad y el conocimiento técnico-científico necesario; 2) un marco jurídicoinstitucional que estipule y defina las competencias y facultades de los órganos subnacionales; 3) la voluntad y respaldo político por parte de gobiernos provinciales; 4) la capacidad institucional de los gobiernos subnacionales; 5) la visión de las autoridades locales para incluir temas ambientales en su agenda paradiplomática; 6) la capacidad financiera; y 7) factores geopolíticos.

Si bien todos estos aspectos deben ser considerados, es la voluntad política y la visión de las autoridades locales en turno, junto con la capacidad institucional de los gobiernos subnacionales, los aspectos que pueden influir en la calidad de la actividad paradiplomática ambiental, afectando decisiones tales como la apertura o clausura de oficinas y/o representaciones de asuntos internacionales así como el nivel de prioridad dado a asuntos como el desarrollo local sustentable, la protección de los recursos naturales, la gestión ambiental y la provisión de bienes públicos regionales/globales. Debe considerarse además la ideología o partido de los gobernadores y presidentes municipales, así como su personalidad y formación académica, que inciden directamente sobre su liderazgo y visión de territorio, en el nivel de participación y la forma en que se conducen las relaciones de los gobiernos subnacionales con el exterior (Schiavon y Velázquez, 2009).

A continuación, se describen dos experiencias de paradiplomacia ambiental que han contribuido con la gobernanza ambiental, a saber: la Unidad Temática de Ambiente y Desarrollo Sostenible de la Red de Mercociudades, el Pacto de Alcaldes contra el Cambio Climático promovido por la Comisión Europea; de igual forma -posteriormente- se hace una breve mención a dos experiencias de cooperación transfronteriza en América Latina: la Mancomunidad Trinacional Fronteriza del Río Lempa en las fronteras de El Salvador, Honduras y Guatemala y el denominado "pasaje de fronteras rojas a fronteras verdes" en el caso de Ecuador-Perú.

\section{Dos experiencias de paradiplomacia ambiental en el marco de la integración regional para fortalecer la gobernanza ambiental: una descripción de experiencias comparadas en el Mercosur y la Unión Europea}

En primer lugar, es importante aclarar que éstas iniciativas cuentan con dos perspectivas distintas. La Red Mercociudades representa un proceso de 
abajo-arriba (bottom up), es decir, surgida por la voluntad e interés de los gobiernos locales del Cono Sur, su Unidad Temática de Ambiente y Desarrollo Sostenible (Utads) representa una oportunidad para favorecer políticas de gobernanza horizontal en la materia. En tanto que el Pacto de Alcaldes contra el Cambio Climático nace desde una perspectiva de arriba-abajo (top down) propuesta por la Comisión Europea.

A cuatro años de la entrada en vigencia del Tratado de Asunción, surge en 1995 un espacio plurilateral de ciudades denominado Red de Mercociudades (Oddone, 2008; Granato y Oddone, 2008). Dentro de los estatutos de la red se fija que las unidades temáticas son las instancias responsables del desarrollo de temas y propuestas de políticas públicas específicas de la Red (artículo 28). Sus facultades consisten en la capacidad de formular y promover políticas comunes a sugerir al Mercosur, desarrollar investigaciones, divulgar experiencias y best practices llevadas a cabo en otras ciudades del mundo, promover eventos de discusión acerca del tema de su responsabilidad y elaborar un banco de datos con la información obtenida a partir de los temas coordinados (artículo 29).

El impacto de las iniciativas subnacionales en la gobernanza ambiental acaba alcanzando mayor empuje cuando los actores subnacionales actúan de manera organizada en mecanismos de cooperación horizontal y redes solidarias. La Utads, creada en 1996, lleva a cabo actividades que estimulan la promoción de acciones y desarrollo de programas y proyectos de desarrollo local sostenible. Las temáticas abordadas se centran en materia medioambiental como en preservación del suelo y la biodiversidad, la prevención y la recuperación de la calidad de los medios acuáticos y atmosféricos y la educación ambiental. La gestión de los residuos sólidos es una de las temáticas prioritarias de trabajo. Han mantenido una actividad estable a lo largo de estos años dando lugar a la mayor participación posible de los actores públicos y privados locales. Esto implica la movilización y el compromiso de los diferentes sectores sociales presentes en un territorio, aunque en la práctica, la participación de las comunidades locales varía mucho según cada experiencia y no es posible identificar un único modelo para todos los territorios.

En el 2012, durante la Conferencia de las Naciones Unidas sobre el Desarrollo Sustentable en la ciudad de Río de Janeiro, se lanzó "Mercociudades hacia Río+20". Bajo la premisa de construir una gobernanza regional por medio de acciones participativas y coordinadas, las Mercociudades establecieron una serie de acuerdos plasmados en el documento "Rio +20 : El rol de las ciudades a 20 años de la Cumbre de la Tierra de Río de Janeiro". En este documento se 
procuró avanzar hacia una gestión ambiental moderna, reconociendo el papel de las ciudades y sus aportes locales para planificar y gestionar, así como establecer una nueva gobernanza en pos del desarrollo sustentable. Asimismo, se incluyó la participación ciudadana y de otras organizaciones en torno al desarrollo sustentable y se procedió al fortalecimiento de las capacidades de las ciudades para impulsar espacios y mecanismos para asegurar el acceso a los recursos, entre otros puntos. Esta iniciativa ha constituido una verdadera acción de paradiplomacia ambiental en materia de cambio climático, que consolidó a Mercociudades en uno de los espacios pioneros en debatir políticas públicas que articulan a gobiernos locales y desarrollo sustentable en la región (Alvariza y Granato, 2014). En este documento también se menciona explícitamente la voluntad de las ciudades de fortalecer sus capacidades hacia una nueva gobernanza ambiental, impulsando espacios y mecanismos que aseguren el acceso a recursos de distinto tipo, tanto desde los gobiernos nacionales como desde las agencias internacionales de cooperación y apoyar la definición de una iniciativa a nivel de Naciones Unidas sobre ciudades sustentables para todos, con inclusión social y desarrollo local. Para la realización de este punto, durante 2013 y 2014, la Utads ha prestado colaboración con la oficina de Naciones Unidas para la prevención de Riegos de Desastres (UNISDR) en la campaña Ciudades Resilientes destinada a mostrar la manera en que los gobiernos locales se preparan ante los embates del cambio climático.

En el 2008, la Comisión Europea presentó la iniciativa del Pacto de los Alcaldes con el objetivo de integrar acciones a nivel regional y local para la consecución de los objetivos delineados en el marco de la Iniciativa "20-20-20". ${ }^{8}$ En este sentido, el punto de partida de la iniciativa implica reconocer que: a) los gobiernos locales y regionales comparten la responsabilidad de la lucha contra el calentamiento global con los gobiernos nacionales y, por lo tanto, deben comprometerse independientemente de los compromisos asumidos por otras partes; b) las ciudades y pueblos son directa o indirectamente responsables (como consecuencia del uso de productos y servicios por la ciudadanía) de más de la mitad de las emisiones de gases de efecto invernadero asociadas al uso de energía en las actividades humanas; c) los compromisos de la UE de reducir sus emisiones -así como los de la comunidad internacional- sólo podrán

\footnotetext{
${ }^{8}$ La Comisión Europea lanzó en enero de 2007 la iniciativa "20-20-20" que establecía tres objetivos claves para el año 2020: 1) una reducción del 20\% de las emisiones de gases de efecto invernadero (GEI) en comparación a los niveles de 1990;2) un incremento de la participación de las fuentes renovables en un $20 \%$ del consumo final de energía; y, 3) una suba del $20 \%$ de la eficiencia energética.
} 
lograrse si son compartidos por los agentes locales interesados, la ciudadanía y sus asociaciones; y d) los gobiernos locales y regionales, en calidad de administraciones más cercanas a la ciudadanía, deben liderar la acción y servir como ejemplo, ya que muchas de las acciones que es necesario desarrollar, en relación a la demanda de energía y las energías renovables, para hacer frente a las alteraciones del clima son competencia de los gobiernos locales o no serían viables sin su apoyo político.

Considerando la dificultad que pueden enfrentar los municipios para disponer de los recursos económicos y técnicos, los países firmantes han diseñado una red que cuenta con la figura de Coordinadores del Pacto, es decir, autoridades nacionales, provincias y regiones destinadas a ofrecer orientación estratégica, cooperación técnica y ayuda financiera a aquellos municipios interesados en participar pero que encuentran obstáculos. Además, han creado la figura de Promotores del Pacto, cuya finalidad es aprovechar las redes de contactos, así como optimizar sus actividades comunicativas a fin de promover el Pacto y dar respaldo al compromiso de los firmantes. Esta alianza, con el propósito de concatenar las acciones locales para alcanzar las metas establecidas - no sólo en el Paquete Energía de la Unión Europea sino también en el Protocolo de Kyoto-, ha brindado a los gobiernos el apoyo necesario para la implementación de políticas destinadas a emplear fuentes de energía renovable, mejorar la eficiencia energética y avanzar en la mitigación de los efectos del cambio climático. Si bien ésta estructura diseñada es arquetípica de un esquema de gobernanza multinivel, tal como ha demostrado Strangis (2012), presenta algunos aspectos críticos como los costes de coordinación entre actores, el respaldo que diferentes actores brindan a la iniciativa y la función de guía de la Comisión Europea.

Sin embargo, la importancia del Pacto de los Alcaldes radica, de acuerdo con Beltrán y Strangis (2014), en que aunque es una iniciativa surgida en el marco de la Unión Europea sus efectos trascienden al ámbito internacional principalmente por tres razones. La primera, porque las acciones desarrolladas para combatir el cambio climático redundan en beneficio del planeta con efectos extrafronterizos. La segunda, dado que el Pacto está abierto a entidades locales de terceros países, no solamente de países vecinos, ultrapasa claramente el ámbito europeo. Y finalmente, porque las acciones desarrolladas a partir del Pacto contribuyen a la salvaguarda del medio ambiente que es un bien protegido por una norma imperativa (Beltrán y Strangis, 2014, p. 298). El Pacto es, por tanto, no sólo un instrumento de la Unión Europea sino de la comunidad internacional en su conjunto. 


\section{Paradiplomacia y gestión ambiental regional: dos ejemplos relevantes en la cooperación transfronteriza de América Latina}

Como se mencionó en el apartado anterior, la paradiplomacia contribuye a la gestión ambiental regional lo que se puede evidenciar con mayor relevancia para las zonas de frontera, dada la proximidad entre los gobiernos subnacionales de los estados colindantes. De igual manera, la paradiplomacia puede apoyar la configuración territorial de los espacios comunes a partir de la definición de su uso social y explotación económica sobre la base de una coordinación de actores, el establecimiento de agendas y políticas comunes, la captura de flujos financieros para la sostenibilidad ambiental de área compartida. La cooperación transfronteriza consolida los lazos de confianza a través de acciones que se desarrollan en el territorio común, mediante la cooperación de programas que requieren de la colaboración estrecha entre las autoridades gubernamentales y la sociedad civil local presentes en ambos lados de la línea fronteriza. Esta modalidad de la cooperación internacional promueve el respeto y la interrelación entre las minorías étnicas y, en última instancia, estimula la concertación política entre las partes (Rhi-Sausi y Oddone, 2009).

La paradiplomacia contribuye con los procesos de gestión ambiental transfronteriza favoreciendo la construcción de bienes públicos, regulando la explotación y uso de los espacios compartidos, generando consensos en materia de protección ambiental y avanzando en la prevención de conflictos socio-ambientales. Este tipo de cooperación se ve mayormente estimulada cuando los territorios comprendidos comparten una infraestructura de conectividad física y cuando se reconoce institucionalmente la participación de los gobiernos subnacionales fronterizos, en cuanto articuladores de los actores locales, como instancia fundamental para una positiva gobernanza ambiental transfronteriza (Rhi-Sausi y Oddone, 2012).

Asimismo, puede observarse que las acciones paradiplomáticas se presentan como una estrategia para estrechar los lazos de cooperación entre los distintos niveles de gobierno, no sólo locales, erigiéndose también como una estrategia política para el desarrollo económico, social y ambiental a escala nacional. Esto facilita una mayor gobernabilidad de los recursos ambientales y el establecimiento de acuerdos para su uso compartido y sustentable. A modo de ejemplo, puede considerarse la experiencia de la Mancomunidad Trinacional Fronteriza del Rio Lempa, conformada por municipios de Honduras, Guatemala y El Salvador, que actualmente desarrolla una serie 
de proyectos en materia de medio ambiente relacionados con la gestión de aguas transfronterizas, el cuidado de bosques y la promoción de la seguridad alimentaria (Oddone et al., 2012). Este es un caso que da muestras de cómo la paradiplomacia genera consensos en materia de proyección ambiental y consolida una buena gobernanza de los recursos naturales en las zonas fronterizas y logra proyectarse a escala transnacional en el marco del Plan Trifinio. ${ }^{9}$

La cooperación transfronteriza puede constituirse también en la base propicia para el desarrollo de estrategias de "diplomacia preventiva", en la que los sistemas nacionales, las instituciones regionales y los acuerdos locales contribuyan en la prevención y disipación de situaciones de conflicto a la vez que estimulen los mecanismos de construcción para la paz y la estabilidad social, económica, ambiental y política ante la eventual ocurrencia de desastres naturales. La construcción de un entorno ambiental protegido en zonas que han sido escenario de conflictos bélicos permite una transición desde las fronteras rojas a las denominadas fronteras verdes. Éste fenómeno ha sido señalado como 'ambientalización de la frontera' y puede ser entendido como estrategias de las ONGs para captar más recursos financieros y aplicar modelos de conservación eficientes (Vázquez, 2009).

A modo de ejemplo de "ambientalización de la frontera" pueden considerarse los casos observados en la frontera paraguayo-boliviana, escenario de la Guerra del Chaco (1932-1935) y en la frontera Ecuador-Perú, escenario donde se desarrolló la Guerra de Cenepa en 1995. De esta forma la frontera evoluciona de un modelo de ocupación y gestión por fortines militares desde finales que se extendió hasta principios de la década de 1990 hacia una frontera "regulada" por las áreas silvestres públicas y privadas de conservación transfronteriza bajo un esquema de gobernanza.

\footnotetext{
${ }_{9}^{9}$ El Tratado del Plan Trifinio es el marco legal que permite la ejecución de programas, proyectos e iniciativas trinacionales, promoviendo la cooperación transfronteriza y el manejo sostenible de los recursos naturales compartidos; definiendo a la región como 'una unidad ecológica indivisible, en la que sólo la acción conjunta de los tres gobiernos podrá dar solución satisfactoria a la problemática de la población del área y al manejo sostenible de sus recursos naturales'. El Tratado delimita el espacio geográfico de acción en el territorio Trinacional y las competencias de los gobiernos en función de la ejecución del mismo; institucionaliza a la Comisión Trinacional del Plan Trifinio conformada por los Vicepresidentes de El Salvador y Guatemala y un Designado a la Presidencia de Honduras. La Comisión cuenta con personalidad jurídica propia, con autonomía administrativa, financiera y técnica, contando con una Secretaria Ejecutiva Trinacional. Es la máxima autoridad de carácter regional Trinacional, encargada de tutelar la ejecución del Plan Trifinio" (Cardona, 2008, p. 261).
} 


\section{Conclusiones}

Los retos que supone el cambio climático impelen la generación de alianzas estratégicas entre actores públicos y privados a diferentes niveles, pues una solución conjunta -en el marco de la gobernanza ambientalresulta mucho más factible y eficiente que acciones individuales. La política ambiental internacional se ha vuelto mucho más compleja por lo que ya no se puede entenderla exclusivamente desde los estados nacionales. La gobernanza ambiental representa un ámbito de acción en el que los gobiernos, no importando cuál sea su nivel (municipal, estatal, provincial, regional o nacional), deben dar respuesta a problemas globales. En este entendido, el vínculo entre paradiplomacia y gobernanza ambiental resulta indisociable dado que la primera contribuye con hacer operativa la segunda.

La importancia de dotar con mayor autonomía a las unidades subnacionales permite que el diseño y la implementación de políticas ambientales sea cada vez más costo-efectivas. En ese sentido, las estrategias (pro)activas de paradiplomacia ambiental pueden sentar las bases para lograr una contribución real de los municipios y gobiernos intermedios a las estrategias nacionales e internacionales de protección ambiental, mitigación y adaptación al cambio climático en el marco de una promoción multinivel de la gobernanza ambiental. Por otro lado, es necesario tener en cuenta que las decisiones y posturas que adoptan la multiplicidad de agentes que intervienen en la gobernanza ambiental pueden presentar opciones y ambigüedades técnicas, económicas y políticas, así como interacciones sociales que oscilan entre relaciones contrapuestas, de cooperativas a conflictivas y de resistencia (de Castro et al., 2015). En este contexto, la paradiplomacia y el papel de los gobiernos locales debe concentrarse en la generación de los consensos claves para la estabilidad de las negociaciones y de las estrategias ambientales de desarrollo.

La paradiplomacia puede significar una ventana de oportunidad para el fortalecimiento de la gobernanza ambiental. Sin embargo, para lograr crear sinergias entre la actividad paradiplomática y las políticas públicas locales, es necesario que los gobiernos subnacionales cuenten con capacidades proyectuales fortalecidas y sean considerados como "autoridades locales responsables" (Oddone et al., 2012), lo que les permita diseñar y ejecutar esquemas de gestión ambiental eficientes. La capacidad de los gobiernos subnacionales de "bajar" la agenda ambiental y de prevención, adaptación y mitigación al cambio climático a los propios territorios y a la sociedad civil local radica en su potencial de convocatoria ciudadana y en la posibilidad 
de diseñar políticas públicas de acuerdo con las preferencias de la población (responsiveness).

Por último, más allá de las experiencias de éxito presentadas en materia de gobernanza ambiental, es importante recalcar que la paradiplomacia representa una oportunidad para abordar las cuestiones de sustentabilidad y adaptación al cambio climático a nivel local pero no es la única fórmula para todas las situaciones o contextos locales. Como herramienta de articulación de intereses y creación de consensos multinivel desde lo bajo, corresponde a las autoridades locales y las fuerzas sociales del presente analizar bajo qué contexto se trata de una herramienta útil.

"Si nos paramos a pensar las pequeñas cosas,

llegamos a entender las grandes."

(José Saramago, Época, Madrid, 21 de enero de 2001)

\section{Referencias}

ALFIE COHEN, Miriam. Introducción. En: Miriam Alfie Cohen (ed.). Comprometidos para negociar: conflicto y gobernanza ambiental (Holanda, Canadá y México). Ciudad de México: Universidad Autónoma Metropolitana y Juan Pablo Editor, 2011. p. 7-25.

ALFIE COHEN, Miriam. Geografías de la gobernanza ambiental: los casos holandés y canadiense. Espacio Abierto, v. 22, n. 1, p. 7-43, 2013.

ALVARIZA, Rafael; GRANATO, Leonardo. Mercociudades y medio ambiente: tendiendo puentes para el desarrollo sostenible. En: Nahuel Oddone; Horacio Rodríguez Vázquez (eds.). Municipios y cambio climático: hacia la construcción de una agenda de paradiplomacia ambiental. Granada: Unión Iberoamericana de Municipalistas. 2014. p. 373-381.

BELTRÁN, Susana; STRANGIS, Davide. La experiencia europea del pacto de alcaldes contra el cambio climático: una impresión desde el derecho internacional. En: Nahuel Oddone; Horacio Rodríguez Vázquez (eds.). Municipios y cambio climático: hacia la construcción de una agenda de paradiplomacia ambiental. Granada: Unión Iberoamericana de Municipalistas. 2014. p. 291-312.

BODANSKY, Daniel. The legitimacy of international governance: a coming challenge for international environmental law? The American Journal of International Law, v. 93, n. 3, p. 596-624, 1999.

BRENNER, Ludger. Gobernanza ambiental, actores sociales y conflictos en las Áreas Naturales Protegidas mexicanas. Revista Mexicana de Sociología, v. 72, n. 2, p. 283-310, 2010.

BULKELEY, Harriet. Reconfiguring environmental governance: towards a politics of scale and networks. Political Geography, v. 24, n. 8, p. 875-902, 2005. 
CARDONA, Rokael. La integración centroamericana desde lo local: La Mancomunidad Trinacional Fronteriza Río Lempa. Anuario 2008. Observatorio de Cooperación Descentralizada Unión Europea-América Latina. Montevideo: OCD UE-AL, 2008. p. 256-275.

CARDOZO FERNANDES REI, Fernando; CUNHA, Kamyla; SETZER, Joana. La paradiplomacia ambiental en la nueva gobernanza ambiental. En: Nahuel Oddone; Horacio Rodríguez Vázquez (eds.). Municipios y cambio climático: hacia la construcción de una agenda de paradiplomacia ambiental. Granada: Unión Iberoamericana de Municipalistas, 2014. p. 87-94.

CORNAGO PRIETO, Noé. Diplomacia, paradiplomacia y redefinición de la seguridad mundial: dimensiones de conflicto y cooperación. En: Francisco Aldecoa Luzárraga; Michael Keating (eds.). Paradiplomacia: las relaciones internacionales de las regiones. Madrid: Marcial Pons, 2001. p. 55-78.

DE CASTRO, Fabio; HOGENBOOM, Bárbara; BAUD, Michiel (eds.). Gobernanza ambiental en América Latina. Buenos Aires: Clacso, 2015.

DECLARACIÓN de Gauteng. 2002 <www.nrg4sd.org/wp-content/uploads/2015/06/ gauteng-es.pdf> (6 jun. 2018).

GRANATO, Leonardo; ODDONE, Nahuel. Mercociudades, red de integración: una nueva realidad en América Latina. Buenos Aires: Capital Intelectual, 2008.

LEEUWIS, Cees; VAN DEN BAN, Anne. Communication for rural innovation: rethinking agricultural extension. Oxford: Blackwell Publishing, 2004.

MACHT, Axel. La gestión de la GTZ (Cooperación Técnica Alemana) para el desarrollo local sustentable en el marco de la descentralización en México. En: Adelaida Esther Ponce Adame; Gabriela Sánchez Gutiérrez (eds.). Cooperación internacional para el desarrollo local: aspectos estructurales, experiencias, oportunidades y limitaciones. Ciudad de México: Instituto Mora y Universidad Autónoma Metropolitana, 2010. p. 113-124.

OOTSVEEN, Irene. El papel de las asociaciones de gobiernos locales en la gestión internacional municipal: hacia una ambición en México. En: Adelaida Esther Ponce Adame; Gabriela Sánchez Gutiérrez (eds.). Cooperación internacional para el desarrollo local: aspectos estructurales, experiencias, oportunidades y limitaciones. Ciudad de México: Instituto Mora y Universidad Autónoma Metropolitana, 2010. p. 204-224.

ODDONE, Nahuel; RODRÍGUEZ VÁZQUEZ, Horacio (eds.). Municipios y cambio climático: hacia la construcción de una agenda de paradiplomacia ambiental. Granada: Unión Iberoamericana de Municipalistas, 2014.

ODDONE, Nahuel; TORRES, Loída; CASTELLANOS, Onilda; GONZÁLEZ, Mirio. Estudio comparado de la legislación municipal de los países que conforman el Sistema de la Integración Centroamericana (Sica) para identificar una base legal que promueva e incentive la integración centroamericana de los gobiernos locales. Ciudad de Guatemala: Mancomunidad Trinacional Fronteriza del Río Lempa y Programa Urbal III, 2012. 
ODDONE, Nahuel. La red de mercociudades: globalización, integración regional y desarrollo local. Valencia: Universidad Politécnica de Valencia, 2008.

PONCE, Esther. Introducción. ¿Por qué hablar de la cooperación internacional para el desarrollo local? Un análisis para la praxis. En: Esther Ponce; Gabriela Sánchez (eds.). Cooperación internacional para el desarrollo local: aspectos estructurales, experiencias, oportunidades y limitaciones. Ciudad de México: Instituto Mora y Universidad Autónoma Metropolitana, 2010. p. 11-26.

PONCE ADAME, Adelaida Esther; SÁNCHEZ GUTIÉRREZ, Gabriela (eds.). Cooperación internacional para el desarrollo local: aspectos estructurales, experiencias, oportunidades y limitaciones. Ciudad de México: Instituto Mora y Universidad Autónoma Metropolitana, 2010.

RHI-SAUSI, José Luis; ODDONE, Nahuel. Cooperación transfronteriza e integración: oportunidades para el desarrollo del Perú. En: MOYA MENA, Sergio (ed.). Las fronteras: espacios de encuentro y cooperación. San José de Costa Rica: Flacso, 2012. p. 19-64.

RHI SAUSI, José Luis; ODDONE, Nahuel. Introducción. En: José Luis Rhi Sausi; Dario Conato (eds.). Cooperación transfronteriza e integración en América Latina. Roma: Centro Studi di Politica Internazionale, 2009. p. 11-31.

RHODES, Rod. Understanding governance. London: Open University Press, 1997.

RODRÍGUEZ VÁZQUEZ, Horacio; LUCATELLO, Simone. Cooperación internacional e inteligencia territorial: apuntes para la construcción de una paradiplomacia ambiental. En: Zidane Zeraoui (ed.). Teoría y práctica de la paradiplomacia. Puebla: Tecnológico de Monterrey, 2013. p. 147-170.

RODRÍGUEZ VÁZQUEZ, Horacio; LUCATELLO, Simone; ODDONE, Nahuel. Paradiplomacia ambiental e inteligencia territorial frente al cambio climático. En: Nahuel Oddone; Horacio Rodríguez Vázquez (eds.). Municipios y cambio climático: hacia la construcción de una agenda de paradiplomacia ambiental. Granada: Unión Iberoamericana de Municipalistas. 2014, p. 95-107.

RUIZ, Juan José. Derecho internacional del medio ambiente. Madrid: Mcgraw-Hill, 1999.

SCHIAVON, Jorge; VELÁZQUEZ FLORES, Rafael. La paradiplomacia de las entidades federativas en México. En: Zidane Zeraoui (ed.). Regionalización y paradiplomacia. La política internacional de las regiones. Puebla: Tecnológico de Monterrey, 2009. p. 65-87.

STRANGIS, Davide. Gobiernos locales y cambio climático: la implementación del Pacto de los Alcaldes - estudio de caso en Italia y España. En: Quaderns de Recerca (Bellaterra). Barcelona: Institut Universitari d'Estudis Europeus, 2012.

VAZQUEZ, Fabricio. D'une périphérie oubliée à des multi-territorialités activées: nouvelles configurations spatiales et économiques dans le Chaco paraguayen. Toulouse: Toulouse II Le Mirail, 2009.

VELAZQUEZ FLORES, Rafael; SCHIAVON, Jorge. Marco normativo e institucional de la cooperación internacional descentralizada de los gobiernos locales en México. 
En: Esther Adelaida Ponce Adame; Gabriela Sánchez Gutiérrez (eds.). Cooperación internacional para el desarrollo local: aspectos estructurales, experiencias, oportunidades y limitaciones. Ciudad de México: Instituto Mora y Universidad Autónoma Metropolitana, 2010. p. 29-57.

Recibido el: 10 ene. 2018

Aprobado el: 26 mar. 2018

Autor correspondiente:

Nahuel Oddone

Calle circunvalación, 294

Colonia San Benito

San Salvador, El Salvador

NAHUEL ODDONE <noddone@cenpromype.org>

Doutor en Estudios Internacionales por la Universidad del País Vasco/Euskal Herriko Unibertsitatea (Bilbao, Espanha), Asesor Regional de Entorno de Negocios del Centro Regional de Promoción de la Mipyme (Cenpromype) del Sistema de la Integración Centroamericana (Sica) (San Salvador, El Salvador)<oddone.nahuel@gmail.com>. ORCID: https://orcid.org/0000-0002-3120-3914

HORACIO RODRÍGUEZ VÁZQUEZ < horacio.rodvaz@gmail.com>

Mestre en Cooperación Internacional para el Desarrollo (Instituto Mora, Ciudad de México, México), coordinador del programa REDD+ en la oficina regional para México y norte de América Central de The Nature Conservancy (Ciudad de México, México)<h.rodriguezvazquez@tnc.org>.

ORCID: https://orcid.org/0000-0002-1184-6445

MARTÍN J. QUIROGA BARRERA ORO <mquirogabarreraoro@gmail.com>

Mestre en Políticas Públicas por la Universidad Torcuato di Tella (Belgrano, Argentina), especialización en Política Económica, Consultor de Vivienda y Desarrollo Urbano, Banco Interamericano de Desarrollo (Washington D.C., Estados Unidos)<martinq@iadb.org>.

ORCID: https://orcid.org/0000-0002-3450-0754 